\title{
Sonification of Astronomical Data
}

\section{Wanda L. Diaz-Merced ${ }^{1}$, Robert M. Candey ${ }^{2}$, Nancy Brickhouse ${ }^{3}$, Matthew Schneps ${ }^{3}$, John C. Mannone ${ }^{4}$, Stephen Brewster ${ }^{1}$, and Katrien Kolenberg ${ }^{3,5}$}

\author{
${ }^{1}$ Glasgow University, Scotland, UK. email: wanda@dcs.gla.ac.uk \\ ${ }^{2}$ NASA, Goddard Space Flight Center, Greenbelt. Maryland, USA \\ ${ }^{3}$ Harvard-Smithsonian Center for Astrophysics, Cambridge, MA, USA \\ ${ }^{4}$ Shirohisa Ikeda Project Gurabo, Puerto Rico \\ ${ }^{5}$ Instituut voor Sterrenkunde, Leuven, Belgium
}

\begin{abstract}
This document presents JAVA-based software called XSONIFY that uses a sonification technique (the adaptation of sound to convey information) to promote discovery in astronomical data. The prototype is designed to analyze two-dimensional data, such as time-series data. We demonstrate the utility of the sonification technique with examples applied to X-ray astronomy and solar data. We have identified frequencies in the CHANDRA X-Ray observations of EX Hya, a cataclysmic variable of the intermediate polar type. In another example we study the impact of a major solar flare, with its associated coronal mass ejection (CME), on the solar wind plasma (in particular the solar wind between the Sun and the Earth), and the Earth's magnetosphere.
\end{abstract}

Keywords. Solar Wind, Sonification, xSonify, Variable Stars

\section{Introduction}

Sonification is a developing field. It integrates a wide variety of professional fields and broadens the interaction between users who are accessing information in a diversity of modalities (see http://www.icad.org, the International Community for Auditory Display). For scientific purposes, it employs the highly developed sense of hearing as an adjunct to data visualization in order to enhance current techniques for data analysis. Since the beginning of spacecraft experiments, it became evident that space plasmas were abundant with numerous kinds of nonlinear plasma waves. The new generation of spacecraft with advanced experimental techniques are forever engaged in an ongoing improvement in accuracy and time resolution to capture plasma, particle, radio, magnetic field, and X-ray data (to mention just a few) with increasing accuracy and time resolution. Developments in both the speed and storage capacity of computers have made it possible to perform numerical simulations with increasingly larger numbers of particles and more grid cells for a longer running time, and with higher phase-space dimensions.

A major problem limiting the utilization of visual displays is that data typically contain much more information than can be displayed effectively with currently available technologies. It is also important to consider the limitations imposed by the nature of the human eye. For example, even the best computer screens available today are limited to a range of spatial resolutions. That limitation affects the useful dynamic range of the display, reducing the amount of data scientists can study at any one time. Scientists currently work around such limitations by filtering the data so as to display only the information which they believe is important to the problem in hand. But since that involves making some guesses about the results they are searching for, many discoveries may be missed. 


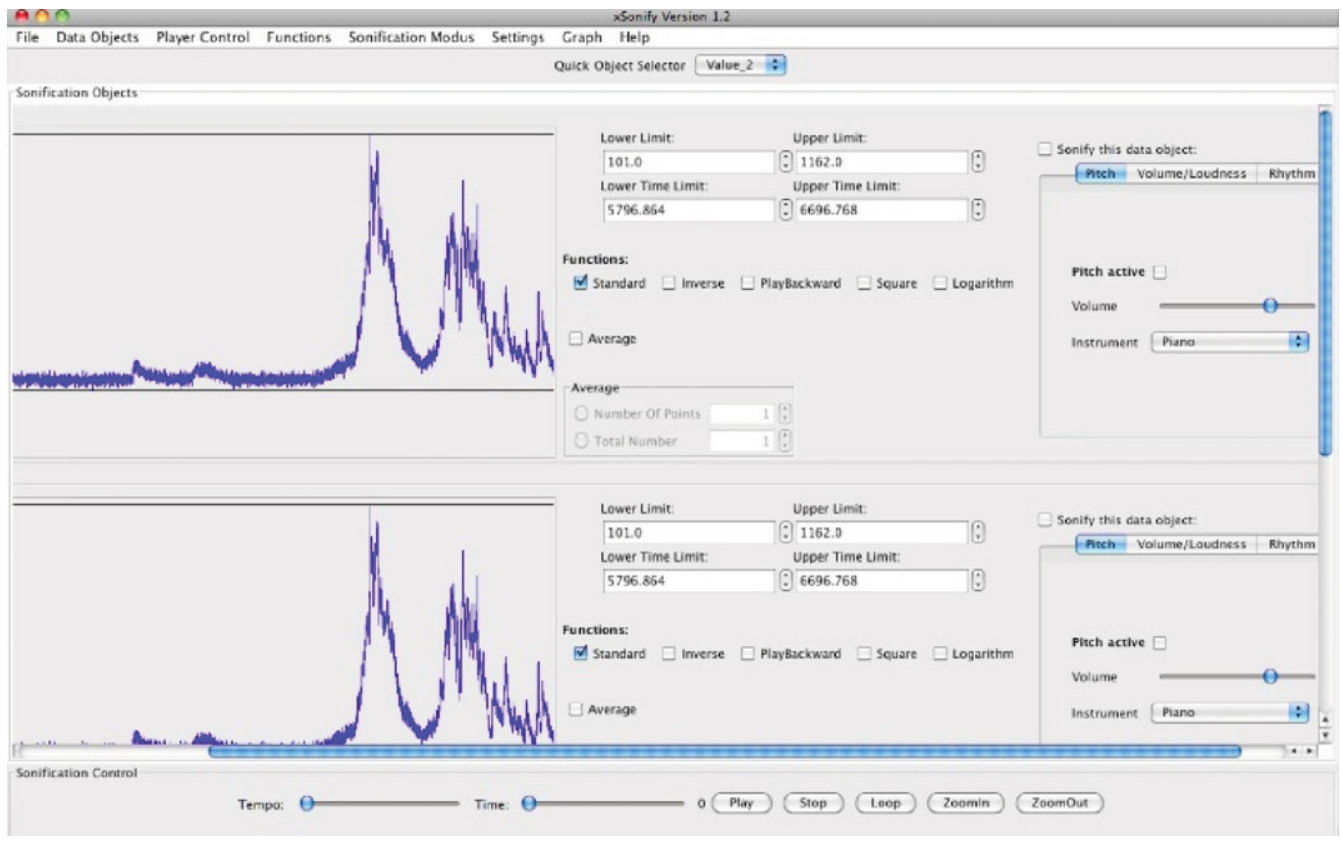

Figure 1. XSONIFY prototype

XSONIFY is a tool for analysing space physics data with an improved functional interphase and allowing a wide variety of file input formats. This tool will open up the SPDF space physics data collection (see http://www.spdf.gsf c.nasa.gov, NASA's Space Physics Data Facility) to a new community of researchers that are currently excluded from space physics research.

Sonification provides other benefits for researchers, including the ability to:

- Analyze complex or rapidly/temporally changing data

- Explore large datasets (particularly multi-dimensional datasets)

- Explore datasets in frequency dimensions rather than spatial ones

- Identify new phenomena which current display techniques miss

- Find correlations and patterns which are masked in visual displays

- Monitor data while doing something else (e.g., background event-finding).

For example, when searching for bow-shock and magnetopause crossings we expect distinctive signatures to be especially apparent above the background noise if the data are sonified. Those boundary signatures appear as characteristic changes in the whole spectrum, including the background noise; other emissions appear as tones at distinctive frequencies or time-frequency spectra, such as electron plasma oscillations, magnetic noise bursts in the magnetosheath, or whistler mode emissions marking regions of currents.

\section{XSONIFY}

XSONIFY sonifies 2-dimensional data from text files and sample files from the CDAWeb heliophysics holdings. It is based on JAVA 1.5 with JAVA Sound, MIDI, JAVASpeech, WebStart and Web Services technology. Currently it features three different sonification modes (Pitch, Loudness, Rhythm) with various controls (Play, Stop, Loop, Speed, Time point) - see Fig. 1. It has limited pre-processing of input data (Limits, Invert, Logarithm, 
Averaging). We will expand it to handle all 2-dimensional plus multi-dimensional data, and add additional necessary features.

The software follows a modular data pipeline approach (Daudé and Nigay, 2003):

- Data Transformation: to appropriate geophysical units;

- Normalization: convert to abstract normalized view;

- Sonification Transformation: to abstract sound parameter space;

- Auditory Display Transformation: sonic rendering.

We have made the source code openly available at http://xsonify . sourceforge.net under the NASA Open Source Agreement (NOSA). As it becomes more stable and capable, we will advertise it widely and develop a community of active users. We have designed an iterative development procedure whereby we solicit requirements and feedback from volunteers (and other interested users) who are evaluating its usability; we then prioritize the requirements according to their importance, usefulness and difficulty of implementation, and produce the next version for further testing.

\section{Examples using XSONIFY}

The X-ray variable EX Hydrae. We sonified time-series of the EX Hya light-curve data in order to analyze the frequency content of the data. The registry space spanned (the relative high or low frequency of discrete or noise sound) can give information on instantaneous frequency changes. The notes are approached as the product of several frequencies (fundamentals, harmonics, etc.). Temporal fluctuation information is then portrayed as a simultaneously sounded cluster of pitches. When each data set was sonified we derived a pitch that changed according to phase, frequency and time variations. The different spans were grouped and then extracted from the data. Those correspond to spin and orbit parameters that have to be extracted from the data to be characterized. Data acquired at a sampling-rate resolution of $1 \mathrm{sec}$ were converted from .FITS to .txt and imported into the sonification prototype. The data were heard in sets of 4,000, with each data set overlapping by ten minutes with the previous one. Two continuous segments thus overlapped by 10 minutes. As a reference to our ears and for the conversion from musical note to data frequency, we calibrated the frequencies in terms of the notes we recognized. A period search by extracting the harmonics using all the data then showed several statistically significant periods in the range between 250 and 800 sec (see Fig. 2).

Solar Wind: ACE, WIND, GOES. We attempted to use sonification techniques to analyze the impact of a major solar flare (X17 Halloween Storm) on the solar plasma, in particular, the solar wind between the Sun and the Earth, and the astrophysical cavity which is the Earth's magnetosphere. The focus was on sonification settings to analyze the X17 flare and its associated halo CME, initiated 2003 October 28. Particle flux and magnetic fluctuation data were extracted from a constellation of satellites: Advanced Composition Explorer (ACE), the WIND space probe, and the Geostationary Operational Environmental Satellite (GOES). The power spectrum was sonified, giving special emphasis to the harmonic content of the sound and restricting the sonification to a window span of 1 decade between $10-1000 \mathrm{mHz}$, detecting frequency-averaged level and descending tones. Those correspond to spectral indices, which in turn tell us something about the physical processes in the plasma. We approached instantaneous changes using sound and/or the notes produced by a timbre in terms of a sum of a number of distinct frequencies such as harmonics, fundamentals, partials, non-harmonics, etc. We listened to satellite time-series data to identify changes in the spectral index of the space plasmas. 


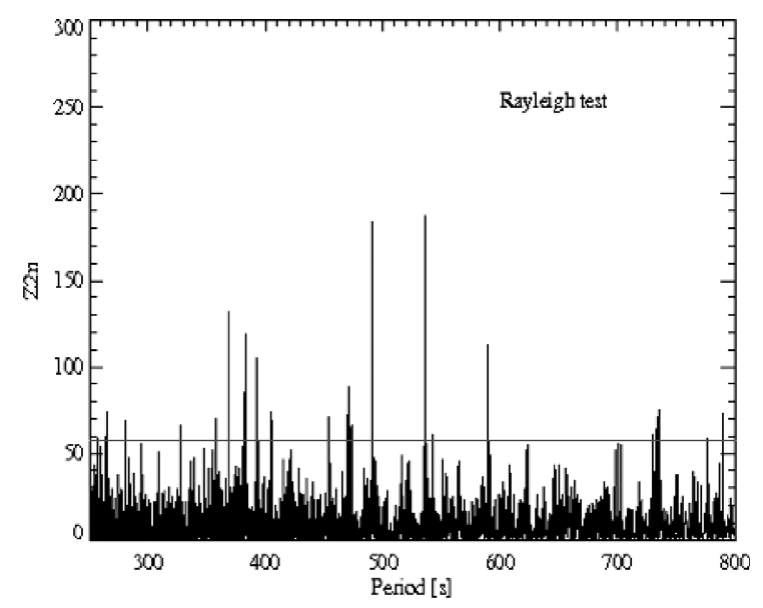

Figure 2. Rayleigh test periodogram, courtesy G. J. M. Luna

The sonification showed both expected and unexpected changes in the power spectra. The sounds were characterized using FFT algorithms.

The GOES satellite data preliminary spectral index -1.20 was not as negative as heard in the sonification. Sonification might indicate events for which a characterization of the descending tones have to be developed. Listening to turbulent data can be done meaningfully in frequency space, and it might also be possible to detect differences in phase velocity. Listening to power spectra may also be meaningful in the frequency space, by restricting the year-to-decade windows between 10-1000 $\mathrm{mHz}$. The descending tones and frequency average levels will correspond to the spectral index.

Direct Mapping Sonification has so far been useful to detect unexpected changes in the data being analyzed (e.g., Kolmogorov power spectra $-5 / 3$ in SwIFT data). Sonification provides an inexpensive and accessible tool to examine the impact of a major solar flare on the solar wind, magnetosphere and ionosphere. It is necessary to develop a threedimensional analysis of fast solar wind and slow solar wind using sound. We use the latter to extract vector components for further correlation.

\section{Conclusions}

Characterization of sounds using Fourier transformations provides a fair approximation to the data. Sonification might indicate local changes that are better characterized with non-stationary data assumptions. Documentation on how to listen to different spacephysics data sets and on sonification techniques will be developed, to extend sonification as a reliable data analysis tool for use by the entire scientific community. Currently, perception experiments are being designed and carried out to demonstrate how sonification techniques may be used as an adjunct to astrophysical data visualization by the sighted.

\section{Acknowledgements}

This research is sponsored by the Smithsonian Women's Committee.

\section{Reference}

Daudé, S. \& Nigay, L. 2003, Proc. ICAD 2003, p. 176 